\title{
Acute Pancreatitis as a Precipitant of Acute-on-chronic Liver Failure in a Patient with Underlying Hepatitis C-related Cirrhosis
}

\author{
${ }^{1}$ Deepak Gunjan, ${ }^{2}$ Vishal Sharma
}

\begin{abstract}
A 66-year-old male, had coronary artery disease and diabetes mellitus, presented with acute onset epigastric pain with radiation to back. He had non-bilious vomiting of 4 days duration. Initial blood investigation showed elevated serum amylase. Abdominal imaging revealed gallstones, edematous pancreatitis with per-pancreatic fat stranding, and evidence of chronic liver disease. Further etiological work-up for chronic liver disease showed anti-hepatitis $\mathrm{C}$ virus (HCV) reactivity. Small varices were detected on upper gastrointestinal endoscopy. During hospitalization, he developed ascites, non-cholestatic jaundice, and hepatic encephalopathy. Ascitic fluid analysis showed evidence of spontaneous bacterial peritonitis and serum-ascites albumin gradient of $1.8 \mathrm{~g} / \mathrm{dL}$. He was treated with intravenous antibiotics and syrup lactulose and discharged with the advice for maintaining salt restricted diet and was initiated on diuretics. Biliary acute pancreatitis acting as a precipitant for acute-on-chronic liver failure (ACLF) is an uncommon event. This case highlights acute pancreatitis as a noninfectious and nonhepatic insult to precipitate ACLF in previously unknown chronic liver disease related to HCV.
\end{abstract}

Keywords: Acute-on-chronic liver failure, Ascites, Cirrhosis, Gallstone, Hepatitis C virus, Pancreatitis.

How to cite this article: Gunjan D, Sharma V. Acute Pancreatitis as a Precipitant of Acute-on-chronic Liver Failure in a Patient with Underlying Hepatitis C-related Cirrhosis. J Gastrointest Infect 2018;8(1):46-47.

Source of support: Nil

Conflict of interest: None

\section{INTRODUCTION}

Acute-on-chronic liver failure (ACLF) is a distinct clinical entity. Various definitions are in vogue for ACLF. It has been defined as acute hepatic insult manifesting as jaundice and coagulopathy, complicated within 4 weeks

\footnotetext{
${ }^{1,2}$ Assistant Professor

${ }^{1}$ Department of Gastroenterology, All India Institute of Medical Sciences, New Delhi, India

${ }^{2}$ Department of Gastroenterology, Postgraduate Institute of Medical Education and Research, Chandigarh, India

Corresponding Author: Vishal Sharma, Assistant Professor, Department of Gastroenterology, Postgraduate Institute of Medical Education and Research, Chandigarh, India, e-mail: docvishalsharma@gmail.com
}

by ascites and/or encephalopathy in a patient with previously diagnosed or undiagnosed chronic liver disease. ${ }^{1}$ Also, it has been purported to include any precipitating event resulting in deterioration of hepatic function and often associated with organ failure. ${ }^{2}$ The causes of acute injury in ACLF are variable, and it can be infectious and noninfectious. ${ }^{1}$ Interestingly, one report ascribes up to $60 \%$ of cases of acute insults in ACLF to be nonhepatic. ${ }^{3}$ We describe here the case of ACLF where acute biliary pancreatitis was the acute insult resulting in presentation with jaundice and ascites in a patient with the previously undiagnosed liver disease because of $\mathrm{HCV}$.

\section{CASE REPORT}

A 66-year-old male, a retired government employee was a known diabetic and had coronary artery disease. He presented with acute onset epigastric pain abdomen with radiation to the back of 4 days duration. The pain was associated with recurrent bouts of non-bilious vomiting. After 2 days of onset of pain, he developed non-cholestatic jaundice and progressive diffuse abdominal distention. On physical examination, he had pallor, icterus, hepatosplenomegaly, and ascites. On investigations, his hemoglobin was $12.8 \mathrm{~g} / \mathrm{dL}$, total leukocyte count $-8000 / \mu \mathrm{L}$, and platelet count-68,000/ $\mu \mathrm{L}$. Liver function tests revealed elevated bilirubin (total bilirubin-9.41 mg/dL, conjugated bilirubin-6.13 mg/dL) and transaminases (aspartate aminotransferase-101 U/L, alanine aminotransferase-135 $\mathrm{U} / \mathrm{L})$, alkaline phosphatase-62 U/L, prothrombin time 30 seconds (control-14 seconds) and amylase- $392 \mathrm{U} / \mathrm{L}$ (normal $<100 \mathrm{U} / \mathrm{L}$ ). Ascites was high as indicated by serum-ascites albumin gradient (SAAG) of $1.8 \mathrm{~g} / \mathrm{dL}$ ) and amylase of $29 \mathrm{U} / \mathrm{L}$. There was evidence of spontaneous bacterial peritonitis with 600 cells per $\mathrm{mm}^{3}$ with neutrophilic predominance. The ascitic fluid cultures were sterile. On etiological workup for chronic liver disease, anti-HCV was reactive. HCV RNA levels were $>10^{6} \mathrm{IU} / \mathrm{mL}$ and the genotype was 3. Ultrasonography of abdomen showed small liver with an irregular outline, splenomegaly, ascites, and cholelithiasis. Contrast-enhanced computed tomography was performed on the abdomen, and it showed evidence of edematous pancreatitis with fat stranding, ascites and nodular liver. Upper gastrointestinal 
endoscopy revealed three columns of small varices. Rest of the workup for liver disease including hepatitis B surface antigen, autoimmune marker, iron profile, and serum ceruloplasmin were normal. He was treated with intravenous ceftriaxone and syrup lactulose. He was discharged with the advice to maintain a salt-restricted diet and was initiated on diuretics. At 4 weeks of followup, he was free of ascites, and his bilirubin had reduced to $2.3 \mathrm{mg} / \mathrm{dL}$.

\section{DISCUSSION}

In ACLF, there is rapid deterioration in a patient with known or unknown chronic liver disease. It is associated with increased mortality and prolongs hospitalization. Both hepatic (acute viral hepatitis) and non-hepatic insults (drugs, alcohol, etc.) can precipitate ACLF likeinfection. ${ }^{1,3}$ ACLF can be complicated by spontaneous bacterial peritonitis, hepatic encephalopathy, jaundice, renal failure, respiratory failure, and hypotension. It has been postulated that systemic inflammatory response (SIRS) leads to a transition from stable cirrhosis to ACLF and pro-inflammatory cytokines are responsible for its various manifestations and organ failures that may accompany ACLF. ${ }^{2}$

Acute pancreatitis is an acute inflammatory condition of the pancreas due to premature activation of proteases within pancreatic acinar cells. Trypsin activation leads to inflammation and production of proinflammatory cytokines resulting in SIRS and organ failures. ${ }^{4}$ Thus, SIRS is a common denominator in both ACLF and acute pancreatitis which leads to disease manifestations. Jaundice can be a feature of acute pancreatitis in cases of choledocholithiasis, cholangitis and common bile duct (CBD) compression due to fluid collections. In index case, there was no evidence of choledocholithiasis, cholangitis or CBD compression. Ascites do occur in acute pancreatitis; however, in our patient ascitic fluid amylase was within normal limits but SAAG was high. Kuo et $\mathrm{al}^{5}$ studied the association of acute pancreatitis with fulminant hepatic failure and acutely decompensated chronic liver disease. These workers found that acute pancreatitis is associated with severe hepatocellular synthetic dysfunction, increased risk of organ failure, more rapid decompensation, and significantly greater mortality. ${ }^{5}$ However, there is no evidence to link the chronic liver disease to the occurrence of biliary pancreatitis. In this case, the patient presented with acute biliary pancreatitis, which precipitated the ACLF in HCV related chronic liver disease and complicated by spontaneous bacterial peritonitis, jaundice, and hepatic encephalopathy.

In conclusion, acute pancreatitis as a precipitant of ACLF is a rare event and is, therefore, being reported. The present report also highlights the fact that non-infectious non-hepatic etiologies must be considered in the differential diagnoses in patients with ACLF where the acute precipitant is uncertain.

\section{REFERENCES}

1. Sarin SK, Kumar A, Almeida JA, Chawla YK, Fan ST, et al. Acute-on-chronic liver failure: consensus recommendations of the Asian Pacific Association for the study of the liver (APASL). Hepatology international. 2009 Mar 1;3(1):269-282.

2. Jalan R, Fernandez J, Wiest R, Schnabl B, Moreau R, Angeli P,et al. Bacterial infections in cirrhosis: a position statement based on the EASL Special Conference 2013. Journal of hepatology. 2014 Jun 1;60(6):1310-1324.

3. Duseja A, Chawla YK, Dhiman RK, Kumar A, Choudhary N, Taneja S. Non-hepatic insults are common acute precipitants in patients with acute on chronic liver failure (ACLF). Dig Dis Sci 2010;55:3188-3192.

4. Banks PA, Freeman ML. Practice guidelines in acute pancreatitis. Am J Gastroenterol 2006;101:2379-2400.

5. Kuo PC, Plotkin JS, Johnson LB. Acute pancreatitis and fulminant hepatic failure. J Am Coll Surg 1998;187:522-528. 